\title{
A Framework for End-to-End QoS Context Transfer in Mobile IPv6*
}

\author{
Chuda Liu ${ }^{1,3}$, Depei Qian ${ }^{1,2}$, Yi Liu ${ }^{1}$, and Kaiping Xiao ${ }^{1}$ \\ ${ }^{1}$ Computer Science Department, Xi'an Jiaotong University, Xi'an, P.R.C, 710049 \\ $\{1 \mathrm{cdm}$, depeiq, liuyi97\}@263. net \\ ${ }^{2}$ School of Computer, Beihang University, Beijing, P.R.C, 100083 \\ ${ }^{3}$ Changsha Aeronautical Vocation Technical College, Changsha, P.R.C, 410124 ,
}

\begin{abstract}
Providing Quality-of-Service (QoS) guarantees and mobility support for Internet devices has become a hot research topic in the Next Generation Internet research, since mobile computing is getting widespread. Context transfers allow better node mobility support, and avoid re-initiation of signaling to and from a Mobile Node (MN). However, Context Transfer Protocol (CTP) [1] proposed by IETF can not meet the need of end-to-end QoS mechanisms because contexts are only transferred between Access Routers (ARs). This paper presents a framework for end-to-end QoS context transfer based on the architecture of F-HMIPv6, which may provide an end-to-end QoS context transfer for real-time applications, therefore they can get promptly the same forwarding process, minimize the handover service disruption, and avoid initiating the endto-end QoS signaling from scratch after an MN performs handovers. The Context Transfer Data message containing the QoS context information, a hop-byhop extension IPv6 option header, is sent from the previous access router (PAR) to the next access router (NAR) via the Mobility Anchor Point (MAP) where old path and new path meet each other. The QoS entities in the nodes between MAP and NAR will be required to check the QoS context information and reserve appropriate resources for MN's sessions and update the new path data in the QoS entities. After successful context transfers, the resources reserved for MN's sessions will be released on the old path. Our scheme may also reduce the signaling overhead and handover latencies by adopting the FHMIPv6 [2] architecture.
\end{abstract}

\section{Introduction}

With the rapid increase of portable devices such as laptops, PDAs, hand-held computers, and a variety of wireless devices, mobile computing applications have become more practical. Real-time services such as Internet telephony, video conferencing, and video-on-demand should be available in the mobile computing environments. It is important for the mobile Internet environment to provide QoS guarantees in the

* This work is supported by the National High-tech Research and Development Program of China, No. 2001AA112120. 
near future. Providing QoS guarantees and mobility support for Internet devices has become a hot research topic in the next generation Internet research. MIPv6 [3] proposed by IETF allows nodes to move from one subnet to another while maintaining the connectivity and on-going connection between $\mathrm{MN}$ and correspondent nodes (CNs). However, the basic Mobile IPv6 protocol does not provide QoS guarantees except the best-effort services. Two different mechanisms have been proposed to provide QoS guarantees in the Internet by the IETF: the Integrated Services (IntServ) [4] based on Resource ReServation Protocol (RSVP) [5] and the Differentiated Services (DiffServ) [6] based on priority levels. These mechanisms may work well in the wired networks but face new challenges in the mobile networks due to the mobility of hosts. Provision of end-toend QoS in mobile networks is more complex than in wired networks mainly due to the user mobility and the constrained bandwidth of the wireless links.

Recently, many investigators have studied the mechanisms of deploying the existing QoS architectures in the mobile wireless networks [7] [10]. However these schemes provide QoS provision by re-initiating signaling from scratch after an MN performs handovers. This may introduce extra signaling overhead and complexity to the protocols, and waste the precious wireless bandwidth (i.e., MRSVP [7] will make advance resource reservations at multiple locations that the MN may possibly visit after handover), resulting in large latency and packet losses to the MN's on-going sessions.

Context transfer protocol (CTP) has been proposed by the Seamoby working group in IETF. This new concept attempts to achieve seamless handovers for Mobile IPv6. Context refers to the information about the current state of a service to be reestablished on a new subnet. For example, in order to reserve the same RSVP recourses as the MN had in the previous subnet after it moves to a new subnet, the corresponding RSVP state information for obtaining the same packet forwarding treatment is called RSVP context. While context transfer refers to the movement of context from one router or network entity to another as a means of re-establishing specific services on a new subnet or collection of subnets, the main motivation of context transfer is to reduce latency and packet losses by avoiding re-initiating signaling to and from the MN [11]. Example features contained in the context are authentication, authorization, and accounting (AAA), header compression, QoS, and security. We will focus on the QoS feature of context transfer in this paper.

As described in [12], context transfer has some advantages, including seamless operation to the MN's traffic flow, bandwidth saving, and less susceptible to errors. However, CTP cannot meet the need of end-to-end QoS requirement because transferring context only at the last hop access router may be insufficient to completely reinitialize the MN's QoS treatment. Some other routers on the path between MN and $\mathrm{CN}$ may also need to be involved [11]. This paper proposes a scheme to provide an end-toend QoS context transfer for real-time applications. The scheme enables the real-time applications to get promptly the same forwarding process, therefore minimizing the handover service disruption and avoiding initiating the end-to-end QoS signaling from scratch when MN performs a handover. The Context Transfer Data (CTD) message containing the QoS context information, a hop-by-hop extension to the IPv6 header, is sent from the previous access router (PAR) to the next access router (NAR) via the Mobility Anchor Point (MAP) where old path and new path meet each other. 
The QoS entities in the nodes between MAP and NAR will check the QoS context information, reserve appropriate resources for MN's sessions, and update the new path data in the QoS entities. After successful context transfers, the resources reserved for MN's sessions will be released on the old path. Our scheme may also reduce the signaling overhead and handover latency by adopting the F-HMIPv6 [2] architecture.

This paper is organized as follows. In section two, we give an introduction to the related protocols. The design goals and assumptions of the proposed scheme are presented in section 3 . In section 4, we propose a framework for end-to-end RSVP context transfer in Mobile IPv6, including the network model and context transfer process and the performance comparison. Finally, we conclude the paper and present the further work.

\section{Related Work}

\subsection{Fast Handover for Hierarchical MIPv6 (F-HMIPv6) [2]}

The F-HMIPv6 protocol intends to combine the Fast Handovers for Mobile IPv6 (FMIPv6) protocol [13] with the Hierarchical Mobile IPv6 Mobility Management protocol (HMIPv6) [14]. This means that the fast handover mechanism will be deployed over the HMIPv6 networks using the F-HMIPv6 protocol. Therefore, the protocol provides the advantages of both schemes, i.e., a seamless handover scheme with less signaling overhead and lower handover latencies. Moreover, the overall handover latency achieved by FMIPv6 will be further reduced because of local location updating in HMIPv6, while in the original FMIPv6, the Home Agent (HA) and CNs are usually far away.

HMIPv6 deals with reducing the amount and latency of signaling between an MN, its HA and one or more CNs by introducing the MAP which is used by the MN as a local HA. Each MN is assigned two Care-of Addresses (CoAs): RCoA (Regional CoA) and LCoA (on-Link CoA). When the MN moves around within a MAP domain, the RCoA can not be changed and only the LCoA need to be changed and registered with the MAP to bind the RCoA and LCoA. Only when the MN moves out of the MAP domain, the RCoA need to be registered to the HA and the MN sends Binding Update (BU) to CNs.

FMIPv6 reduces packet loss by providing fast IP connectivity as soon as a new link is established. It uses the Layer 2 (L2) triggers to obtain the link address and subnet prefix information of the new attachment point of an MN when it is still connected to PAR. This may reduce movement detection latency of the MN. Through the necessary messages exchanging, the MN may pre-configure the New CoA (NCoA) to reduce the NCoA configuration latency. Moreover, in FMIPv6, the data packets arriving the Previous CoA (PCoA) can be forwarded to the NAR using the tunnel established between the PAR and the NAR.

In F-HMIPv6, the MAP performs the tunneling for fast handover instead of the PAR. By the F-HMIPv6 scheme, the data packets sent by $\mathrm{CN}$ will be tunneled by the MAP toward the NAR during the handover. 


\subsection{Context Transfer Protocol (CTP) [1]}

As mentioned in RFC3374 [11], CTP enables authorized context transfers. The key goals are to reduce latency, minimize packet losses and avoid re-initiation of signaling to and from $\mathrm{MN}$.

The context transfer can be initiated by the mobile node (mobile-controlled) or by the network (network-initiated). The CTP typically operates between the source node (i.e., PAR) and the target node (i.e., NAR). PAR transfers contexts under two general scenarios. In the first scenario, either the MN sends the CT Activate Request (CTAR) to PAR to initiate context transfer, or a Layer 2 trigger triggers the PAR. In response PAR transmits a CTD message that contains feature contexts to NAR. In the second scenario, NAR sends a CT Request (CTR) message to PAR as a result of either receiving the CTAR message sent by the MN or an internal trigger. In response to the CTR message, PAR transmits a CTD message that includes the MN's feature contexts. When receiving a CTD message, NAR may generate a CTD Reply message to report the status of processing the received contexts.

\subsection{QoS-Conditionalized Binding Update in Mobile IPv6 (QCBU) [15]}

QCBU is a QoS-Conditionalized solution that is based on the HMIPv6. When a handover takes place, a QoS option, i.e., a hop-by-hop option header defined in [16], is piggybacked in the BU message and sent to the MAP. Each node between MN and MAP forwards the QoS need contained in the QoS option and checks for the resource availability in the node. If the resource is insufficient, the message is dropped and a negative acknowledgement is sent back to $\mathrm{MN}$. Otherwise the message is forwarded to the next hop. When BU carrying the QoS option finally arrives MAP (the crossover point of the old path and the new path) and MAP satisfies the resource requirement, MAP sends Binding Acknowledgement (BA) back to MN.

Accordingly, a handover takes place only when sufficient resources are available in all nodes along the new transmission path. It also enables MN to choose flexibly among a set of available access points so that $\mathrm{MN}$ can transmit packets through a route that offers satisfied QoS. In addition, the scheme is built upon the hierarchical mobile IPv6 protocol to localize mobility management.

\section{The Design Goals and Assumptions}

Our scheme is based on the following goals and assumptions:

1) The key objective is to construct an effective end-to-end QoS context transfer framework, which may establish QoS context information on each new added routers on the path between the crossover router (where the old and new path meet) and NAR during the MN handover. The real-time applications can obtain the same QoS forwarding treatment as that they had before the handover.

2) The scheme should release any QoS state along the old packet path when contexts are transferred successfully after handover. 
3) The scheme should achieve seamless handover and reduce the signaling overhead and handover latency to minimize service disruption of real-time applications during the handover period.

4) We assume that most handovers take(s) place locally, and we may deploy localized mobility management solution to make the MN's handover transparent to its HA and to CNs with which it is communicated.

5) The scheme operates entirely within the administrative domain of the wireless network, any handover out of the domain is beyond our scope, because the operation policies and the Service Level Agreements (SLAs) need to be renegotiated, so re-establishment or re-negotiation of the level of service would be the preferred case proposed in RFC 3374 [12].

6) Each QoS entity in the routers between PAR-MAP-NAR (shown in Fig. 1) is required to check the QoS context information contained in the CTD message. How the QoS entity processes the QoS context information contained in CTD message is described in section 4.2.1 (QoS entity is defined in [16]).

7) MAP is the crossover point where the old path and the new path meet each other after an MN performs handovers, and can be discovered by using MAP discovery algorithm in HMIPv6.

8) QoS should be supported for both uplink (from MN) and downlink (to $\mathrm{MN}$ ) traffic.

\section{Proposed Solution}

In this section, we firstly show the network reference model. Then the implementing details are described. The performance analysis of the scheme is discussed in the finally.

\subsection{Network Reference Model}

Fig. 1 illustrates the network reference model of the framework for the end-to-end QoS context transfer. The MAP is the crossover point where the old path and new path meet each other after MN performs handovers, which can be discovered by using MAP discovery algorithm in HMIPv6. IR is an intermediate router. When the MN moves from AR1 to AR2, the MAP is located at location (1); while from AR2 to AR3, the MAP is located at location (2).

\subsection{The Framework for End-to-End QoS Context Transfer}

We present an end-to-end QoS context transfer framework to overcome the drawback of the CTP in which contexts are transferred only between the PAR and NAR. In this section, we firstly give the processing rules of the CTD message in the framework, and the details of the end-to-end QoS context transfer are described successively. 


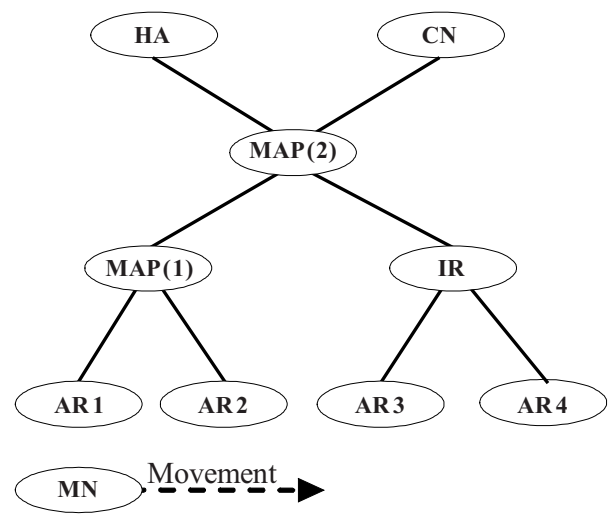

Fig. 1. Network reference model

\subsubsection{The Processing Rules of the CTD Message in the Framework}

In order to implement the end-to-end QoS context transfer, the CTD message is sent from PAR to NAR via MAP. The QoS entity of the router on the path between PAR and NAR is required to check the QoS context information in the CTD message based on the following rules:

1) If the QoS context information carried in the CTD message is found to be the same as the QoS states of the QoS entity in the router, the router simply forwards the CTD message to the next hop without referencing to the QoS context.

2) Otherwise, the router will extract the QoS context information from the CTD message. According to the QoS context received from the CTD message, the router will reserve corresponding resources and update the path information in the QoS entity. For example, the RSVP_HOP object of RSVP needs to be updated when the path has been changed. And the router will send a CTD Reply (CTDR) to PAR to report the status of processing the received contexts. Finally, the CTD message is forwarded to the successive router.

Therefore, when the CTD message is transmitted on the old path, that is, between PAR and MAP, routers just forward the message to the next hop. But when it is transmitted on the new path between MAP and NAR, routers on the path will extract the QoS context and reserve corresponding resources, so the corresponding QoS states are established on the new path.

Consequently, the QoS feature context information is carried in the CTD message as an IPv6 hop-by-hop option in our scheme so that each node on the path can check and process the message individually.

There are two methods to release the QoS setting in the router along the old path if contexts are transferred successfully. One is time-out, i.e., the QoS state is deleted if no refresh message arrives before the expiration of a "cleanup timeout" interval. Another way is to do it explicitly by sending a "teardown" message from MAP to PAR when the CTD Reply (CTDR) message is received by the QoS entity in MAP, for example, in RSVP, RSVP Daemon in MAP will send a "Resv_Tear" message to 
delete the QoS state explicitly along the old path towards the MN. The second method is adopted in this paper.

\subsubsection{The End-to-End QoS Context Transfer Framework}

As mentioned in section 3, when the MN performs a handover, the F-HMIPv6 architecture is employed to achieve a seamless handover, while the end-to-end QoS context transfer is performed using our framework. We will describe the details as the follows.

1) The seamless handover

In order to reduce the handover latency, packet losses, and signaling overhead, the F-HMIPv6 protocol is employed. When the MN undergoes handover, the scheme will follow the processing procedure of the F-HMIPv6 protocol [2] to achieve the seamless handover.

2) The end-to-end QoS context transfer

While the F-HMIPv6 protocol achieves the seamless handover, the end-to-end QoS context transfer is implemented as follows:

- The end-to-end QoS context transfer may be initiated by MN (MN may send the CTAR message to PAR to request contexts transfer) or by the network (PAR starts the transfer of contexts (source trigger) or NAR sends the CTR message to PAR to request contexts (target trigger)).

- The PAR sends the CTD message that includes the QoS context to the NAR via the MAP in response to the context transfer trigger. Each router on the path from PAR to NAR via MAP processes and forwards the CTD message according to the CTD message processing rules described in section 4.2.1.

- When a router on the old path between PAR and MAP receives the CTD message, the QoS entity in the router checks the QoS context information contained in the CTD message. If the QoS context information in the CTD message is the same as that existing in the QoS entity, the router simply forwards the CTD message to the next hop;

- When a router on the new path between MAP and NAR receives the CTD message, the QoS entity in the router checks the QoS context information carried in the CTD message. Because the QoS context information in the CTD message does not exist in the QoS entity, the router extracts the QoS context from the message. If the QoS context information is transferred successfully, the router will reserve corresponding resources and update the path information in the QoS entity. It then forwards the message to the next hop. Otherwise, a CTDR message is sent from the router to PAR to report the failure of the QoS context transfer. In failure case, the CTD message will not be forward to the next node.

- When the NAR successfully processes the received QoS context, the "S" bit in the CTDR message sent by NAR to PAR will be set to one. This may trigger the QoS entity of the MAP to send a Teardown message toward the PAR to delete the QoS states on the old path. 


\subsection{The Performance Analysis and Discussion}

In this section, we compare the performance of our scheme to the QoS solution requirements for Mobile IP [17].

1) Performance requirements

- Our scheme minimizes the interruption in QoS at the time of handover, because an end-to-end QoS context transfer scheme is adopted to allow better support for node mobility, and avoid re-initiating signaling to and from the MN.

- Our scheme localizes the QoS (re) programming to the affected parts of the packet path in the network, since the end-to-end QoS context transfer is performed only between the MAP and the NAR. The MAP is the cross point of the old path and the new path.

- Our scheme provides means to release any QoS state along the old path. If all routers transfer the QoS context successfully, the QoS entity of the MAP will send a Teardown message toward the PAR to delete the QoS status on the old path.

2) Interoperability requirements

- Our scheme may be better interoperable with other mobility protocols because our scheme is based on the F-HMIPv6 protocol which combines the HMIPv6 protocol and FMIPv6 protocol, two protocols that have drawn more attention in the Mipshop working group of IETF.

- Our scheme may interoperate with heterogeneous QoS paradigms such as IntServ and DiffServ, since the QoS context information retrieved from PAR can be better encoded to work with these heterogeneous QoS paradigms.

3) Miscellaneous requirements

- Our scheme can't support QoS along multiple packet paths. This is an open issue and requires further study.

- Our scheme can't provide information to link layer to support required QoS. This needs additional investigation too.

4) Standard requirements

- Scalability: With the deployment of the QoS mechanisms in the network, the QoS states are maintained in the routers by the QoS mechanism itself. Hence, this scheme does not introduce any new scalability issues.

- Security: The security issue in this scheme is as well as that of the CTP. The details are described in section 6 of the CTP [1].

- Conservation of wireless bandwidth: Re-establishing multiple contexts over an expensive, low-speed link can be avoided by relocating contexts over a potentially higher-speed wire [12]. Hence, the scheme may save the precious wireless bandwidth.

- Low processing overhead on mobile terminals: In our scheme, MN needs no additional operations.

- Providing hooks for authorization and accounting: This needs further study.

- Robustness against failures of any Mobile IP-specific QoS components in the network: This needs further study. 


\section{Conclusion and the Future Work}

This paper proposes a framework for end-to-end QoS context transfer in Mobile IPv6 based on the F-HMIPV6 architecture, which may provide lower handover latency and packet losses and less signaling overhead handover, and implement an end-to-end QoS context transfer. The scheme overcomes the weakness of the CTP which can not meet the requirement of the end-to-end QoS mechanisms because of transferring contexts only between PAR and NAR in the CTP. We present the design goals and assumptions of the framework and also provide implementation details of the scheme in this paper. In addition, we compare the performance with scheme of the reinitiating RSVP signaling to re-establish QoS in Mobile IPv6, and show that our scheme has the less latency and packet loss than the scheme.

The simulation based on NS2 [18] platform for the scheme will be done soon to achieve the further performance analysis and discussion. And our further work will be to investigate the way of retrieving, classifying, encoding and representing of the QoS context transfer. The implementation of the end-to-end QoS context transfer framework will be based on the IntServ model, namely we will investigate the end-to-end RSVP context transfer in our further study.

\section{References}

1. J. Loughney (Ed.), M. Nakhjiri, C. Perkins, R. Koodli: Context Transfer Protocol. Internet Draft, draft-ietf-seamoby-ctp-08.txt, work in progress

2. Hee Young Jung, Seok Joo Koh, Hesham Soliman, Jun Seob Lee, Karim El-Malki, Bryan Hartwell: Fast Handover for Hierarchical MIPv6 (F-HMIPv6). Internet Draft, draft-jungmobileip-fastho-hmipv6-02.txt, work in progress

3. D. Johnson, C. Perkins, J. Arkko: Mobility Support in IPv6. Internet Draft, draft-ietfmobileip-ipv6-24.txt, work in progress

4. R. Braden, D. Clark, and S. Shenker: Integrated services in the Internet architecture: an overview. RFC 1633, IETF, June 1994

5. R. Braden, L. Zhang, S. Berson, S. Herzog, and S. Jamin: "Resource Reservation Protocol (RSVP) - Version 1 Functional Specification”. RFC 2205, IETF, September 1997

6. S. Blake, D. Black, M. Carlson, E. Davies, Z. Wang, and W. Weiss: An architecture for differentiated services. RFC 2475, December 1998

7. Talukdar A. K., Badrinath B. R., Acharya A.: MRSVP: A Resource Reservation Protocol for An Integrated Services Network with Mobile Hosts. Wireless Networks, 2001, 7(1): 519

8. S. Paskalis, A. Kaloxylos, E. Zervas: An Efficient QoS Scheme for Mobile Hosts. Workshop on wireless Local Networks (Tampa Florida), November 2001

9. Q. Shen, W. Seah, A. Lo H. Zheng M. Greis: Mobility Extensions to RSVP in an RSVPMobile IPv6 Framework. Internet Draft, IETF, draft-shen-nsis-rsvp-mobileipv6- 00.txt, July 2002

10. Mahadevan I, Sivalingam K. M.: Architecture and Experimental Framework for Supporting QoS in Wireless Networks Using Differentiated Services. Mobile Networks and Applications, 2001, 6(4):385-395 
11. J. Kempf, (Ed.): Problem Description: Reasons for Performing Context Transfers. RFC3374, IETF, September, 2002

12. R. Koodli, C. Perkins: A Context Transfer Protocol for Seamless Mobility. Internet Draft, Draft-koodli-seamoby-ct-04.txt, 2002.8

13. R. Koodli (Ed.): Fast Handovers for Mobile IPv6. Internet Draft, draft-ietf-mobileip-fastmipv6-08.txt, work in progress

14. H. Soliman, C. Castelluccia, K. El-Malki, L. Bellier: Hierarchical Mobile IPv6 mobility management. Internet Draft, draft-ietf- mobileip-hmipv6-08.txt, work in progress

15. A. Festag, X. Fu, H. Karl, G. Schaefer, C. Fan, C. Kappler, M. Schramm, Siemens A. G.: QoS-Conditionalized Binding Update in Mobile IPv6. Internet Draft, draft-tkn-mobileipqosbinding-mipv6-00.txt, July 2001

16. H. Chaskar, R. Koodli: A Framework for QoS Support in Mobile IPv6. Internet Draft, draft-chaskar-mobileip-qos-01.txt, March 2001

17. H. Chaskar, (Ed.): Requirements of a QoS solution for Mobile IP. RFC 3583, IETF, September 2003

18. The Network Simulator NS2: http://www.isi.edu/nsnam 